\title{
ON GREEN'S FUNCTIONS IN THE THEORY OF HEAT CONDUCTION*
}

\author{
H. S. CARSLAW AND J. C. JAEGER
}

1. Introduction. In this Bulletin (vol. 44 (1938), p. 125) Lowan discusses the Green's function for a line source at $\left(r^{\prime}, \theta^{\prime}\right)$ for the cases where the solid (i) is an infinite cylinder $r=a$, and (ii) is bounded internally by $r=a$, radiation taking place at $r=a$ into a medium at zero temperature.

He uses the method of the Laplace transformation. The solution for the first problem agrees with that already obtained by contour integration. $\dagger$ There are some obvious misprints in his discussion of the second problem, and in his solution on page 133 he seems to have used as boundary condition $G=0$ on $r=a$, instead of $\partial G / \partial r+h G=0$, in his notation. His result on that page should read

$$
\begin{aligned}
G=u+v & =\frac{1}{4 \pi} \sum_{n=-\infty}^{\infty} \cos n\left(\theta-\theta_{0}\right) \int_{-\infty}^{\infty} \alpha e^{-k \alpha^{2} t} \frac{H_{n}^{(1)}\left(\alpha r_{0}\right)}{U_{n}(\alpha a)} \\
& \cdot\left\{J_{n}(\alpha r) U_{n}(\alpha a)-H_{n}^{(1)}(\alpha r)\left[\alpha \frac{d}{d z} J_{n}(z)+h J_{n}(z)\right]_{z=\alpha a}\right\} d \alpha,
\end{aligned}
$$

where

$$
U_{n}(\alpha a)=\left[\alpha \frac{d}{d z} H_{n}^{(1)}(z)+h H_{n}^{(1)}(z)\right]_{z=\alpha a} .
$$

Put in this form it can be reduced to the simpler form given below in (16), except for the difference in the sign of $h$.

In this paper we discuss this second problem, first by contour integration, and second by the Laplace transform. In the latter we use what appears to us a much simpler notation and a more rapid approach to the solution.

We remark also that we have used this notation and method in a number of other questions $\ddagger$ and believe that it will be found increasingly useful and much simpler than the operational methods,

* Presented to the Society, September 6, 1938.

$\dagger$ Carslaw, Conduction of Heat, 2d edition, 1921, $\S 888,89$. This book will be referred to below as C.H.

¥Cf. Carslaw, Operational methods in mathematical physics, Mathematical Gazette, vol. 22 (1938), pp. 264-280; Carslaw and Jaeger, Some problems in the mathematical theory of the conduction of heat, Philosophical Magazine, (7), vol. 26 (1938), pp. 473-495. 
due to Heaviside and Bromwich, expounded in Jeffreys' well known tract.*

2. Solution using contour integration. We take the line source at $\left(r^{\prime}, 0\right)$, and we require the solution of the equation

$$
\frac{\partial v}{\partial t}=k\left(\frac{\partial^{2} v}{\partial r^{2}}+\frac{1}{r} \frac{\partial v}{\partial r}+\frac{1}{r^{2}} \frac{\partial^{2} v}{\partial \theta^{2}}\right), \quad t>0, r>a,
$$

which shall tend to zero when $t \rightarrow 0$ in $r>a$, except at $\left(r^{\prime}, 0\right)$, where it is to be infinite as

$$
(4 \pi k t)^{-1} \exp \left\{-\left(r^{2}+r^{2}-2 r r^{\prime} \cos \theta\right) / 4 k t\right\} .
$$

Also, when $r \rightarrow a, v$ is to satisfy

$$
-\frac{\partial v}{\partial r}+h v=0
$$

Let

$$
u=(4 \pi k t)^{-1} \exp \left\{-\left(r^{2}+r^{\prime 2}-2 r r^{\prime} \cos \theta\right) / 4 k t\right\} .
$$

Put $v=u+w$. Then $w$ is to satisfy

$$
\begin{array}{rrr}
\frac{\partial w}{\partial t}=k\left(\frac{\partial^{2} w}{\partial r^{2}}+\frac{1}{r} \frac{\partial w}{\partial r}+\frac{1}{r^{2}} \frac{\partial^{2} w}{\partial \theta^{2}}\right), & t>0, r>a, \\
\lim _{t \rightarrow 0} w=0, & r>a, \\
-\frac{\partial w}{\partial r}+h w=\frac{\partial u}{\partial r}-h u, & r=a, t>0 .
\end{array}
$$

It is known $\dagger$ that

$$
\begin{array}{rlrl}
u & =\frac{1}{2 \pi} \sum_{n=-\infty}^{\infty} \cos n \theta \int_{0}^{\infty} \alpha e^{-k \alpha^{2} t} J_{n}\left(\alpha r^{\prime}\right) J_{n}(\alpha r) d \alpha \\
& =-\frac{1}{4 \pi} \sum_{n=-\infty}^{\infty} \cos n \theta \int \alpha e^{-k \alpha^{2} t} J_{n}\left(\alpha r^{\prime}\right) H_{n}{ }^{(1)}(\alpha r) d \alpha, & r>r^{\prime}, \\
& =-\frac{1}{4 \pi} \sum_{n=-\infty}^{\infty} \cos n \theta \int \alpha e^{-k \alpha^{2} t} J_{n}(\alpha r) H_{n}^{(1)}\left(\alpha r^{\prime}\right) d \alpha, & & r<r^{\prime},
\end{array}
$$

the integrals being taken over the path $P$ of Fig. 1 in the $\alpha$-plane.

* Operational Methods in Mathematical Physics, Cambridge Tracts in Mathematics and Mathematical Physics, no. 23.

$\dagger$ C.H., pp. 184-185. 
For the path at infinity on the right the argument of $4 \alpha$ is less than $\pi$, and on the left it is greater than $3 \pi$. Let

$$
4 \pi w=\sum_{n=-\infty}^{\infty} \cos n \theta \int \alpha e^{-k \alpha^{2} t} A_{n}(\alpha) H_{n}^{(1)}\left(\alpha r^{\prime}\right) H_{n}^{(1)}(\alpha r) d \alpha,
$$

where $A_{n}(\alpha)$ is to be chosen so that $w$ satisfies (7). Thus, using (10), we have

$$
A_{n}(\alpha)\left[\alpha H_{n}^{(1) \prime}(\alpha a)-h H_{n}^{(1)}(\alpha a)\right]=\alpha J_{n}^{\prime}(\alpha a)-h J_{n}(\alpha a) .
$$

Also

$$
4 \pi v=\sum_{n=-\infty}^{\infty} \cos n \theta \int \alpha e^{-k \alpha^{2} t} H_{n}^{(1)}\left(\alpha r^{\prime}\right)\left[A_{n}(\alpha) H_{n}^{(1)}(\alpha r)-J_{n}(\alpha r)\right] d \alpha,
$$

when $r<r^{\prime}$, and we interchange $r$ and $r^{\prime}$ when $r>r^{\prime}$.

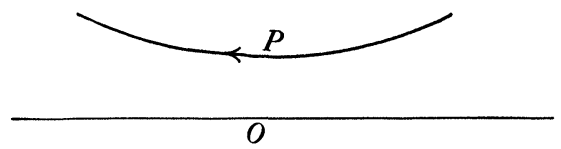

FIG. 1

This value of $v$ satisfies (1) and (3). We shall now show that it satisfies (2); in other words, we shall show that $\lim _{t \rightarrow 0} w=0$, when $r>a$.

The integral in (11) is continuous when $t \geqq 0$. We show that it vanishes when $t=0$. Take the closed circuit of Fig. 2, consisting of

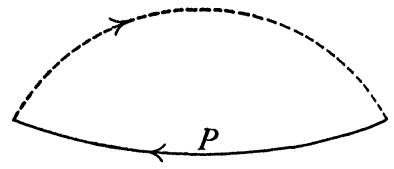

FIG. 2

the path $P$ and the part of a circle, center at the origin, lying above the path $P$.

There are no zeros of $\alpha H_{n}^{(1) '}(\alpha a)-h H_{n}^{(1)}(\alpha a)$ for which the imaginary part of $\alpha$ is positive or zero.* Further the asymptotic expansions for the Bessel functions show that the integral

\footnotetext{
* See the footnote to $\S 6$.
} 


$$
\int \alpha H_{n}^{(1)}(\alpha r) H_{n}^{(1)}\left(\alpha r^{\prime}\right) A_{n}(\alpha) d \alpha
$$

over the circular arc above $P$ tends to zero when the radius tends to infinity. It follows that the integral over the path $P$ tends to zero. We have thus in (14) obtained a solution in the form of an integral over the path $P$.

3. Consider the closed circuit of Fig. 3, formed by the real axis, the path $P$, and the arcs of a circle with center at the origin and radius $R$ (which will tend to $\infty$ ) joining the real axis and the path $P$.

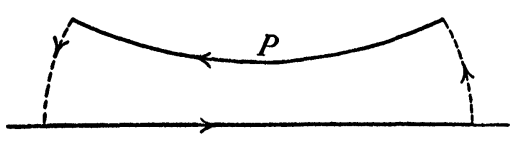

FIG. 3

When $t>0$, the integral of $\S 2,(13)$,

$$
\int \alpha e^{-k \alpha^{2} t} H_{n}^{(1)}\left(\alpha r^{\prime}\right)\left[A_{n}(\alpha) H_{n}^{(1)}(\alpha r)-J_{n}(\alpha r)\right] d \alpha
$$

over the circular arcs tends to zero when $R \rightarrow \infty, r$ being less than $r^{\prime}$. Also there are no poles of the integrand inside this circuit. It follows that the integral over the path $P$ is equal to minus the integral from $-\infty$ to $\infty$ over the real axis. Thus we have

$$
4 \pi v=\sum_{n=-\infty}^{\infty} \cos n \theta \int_{-\infty}^{\infty} \alpha e^{-k \alpha^{2} t} H_{n}^{(1)}\left(\alpha r^{\prime}\right)\left[J_{n}(\alpha r)-A_{n}(\alpha) H_{n}^{(1)}(\alpha r)\right] d \alpha
$$

when $r<r^{\prime}$; and we have to interchange $r$ and $r^{\prime}$ when $r>r^{\prime}$.

4. If we break up $\int_{-\infty}^{\infty}$ into $\int_{-\infty}^{0}$ and $\int_{0}^{\infty}$ and use the formulas

$$
\begin{aligned}
H_{n}^{(1)}(x) & =J_{n}(x)+i Y_{n}(x), \\
H_{n}^{(1)}(x) & =J_{n}^{\prime}(x)+i Y_{n}^{\prime}(x), \\
e^{i n \pi} H_{n}^{(1)}\left(x e^{i \pi}\right) & =H_{n}^{(1)}(x)-2 J_{n}(x)=-J_{n}(x)+i Y_{n}(x), \\
e^{i n \pi} H_{n}^{(1)}\left(x e^{i \pi}\right) & =J_{n}^{\prime}(x)-i Y_{n}^{\prime}(x),
\end{aligned}
$$

the result in $\S 3,(15)$, reduces to

$$
\begin{aligned}
v= & \frac{1}{2 \pi} \sum_{n=-\infty}^{\infty} \cos n \theta \\
& \int_{0}^{\infty} \alpha e^{-k \alpha^{2} t} \frac{U_{n}(\alpha r) U_{n}\left(\alpha r^{\prime}\right)}{\left[\alpha J_{n}^{\prime}(\alpha a)-h J_{n}(\alpha a)\right]^{2}+\left[\alpha Y_{n}^{\prime}(\alpha a)-h Y_{n}(\alpha a)\right]^{2}} d \alpha
\end{aligned}
$$


where

$U_{n}(\alpha r)=J_{n}(\alpha r)\left[\alpha Y_{n}^{\prime}(\alpha a)-h Y_{n}(\alpha a)\right]-Y_{n}(\alpha r)\left[\alpha J_{n}^{\prime}(\alpha a)-h J_{n}(\alpha a)\right] ;$ and this, being symmetrical in $r$ and $r^{\prime}$, holds both for $r<r^{\prime}$ and $r>r^{\prime}$.

5. Solution using the Laplace transformation. We start from equations (1) to (7) of $\$ 2$; by equation (8), $u$ is given in the form $u=$ $\sum_{n=-\infty}^{\infty} u_{n} \cos n \theta$; so we seek a solution of type

$$
v=u+w=\sum_{n=-\infty}^{\infty}\left(u_{n}+w_{n}\right) \cos n \theta
$$

where

$$
\begin{array}{cc}
\lim _{t \rightarrow 0} w_{n}=0, & r>a, \\
\frac{\partial^{2} w_{n}}{\partial r^{2}}+\frac{1}{r} \frac{\partial w_{n}}{\partial r}-\frac{n^{2}}{r^{2}} w_{n}-\frac{1}{k} \frac{\partial w_{n}}{\partial t}=0, &
\end{array}
$$

and the boundary condition (3) is satisfied by $\left(u_{n}+w_{n}\right)$. Now apply the Laplace transformation, using $\bar{u}, \bar{v}, \cdots$ for the Laplace transforms of $u$, $v$, and so on. Thus, ${ }^{*}$ writing $q=(p / k)^{1 / 2}$, we have

$$
\begin{aligned}
\bar{u}=\int_{0}^{\infty} e^{-p t} u d t & =\frac{1}{4 \pi k} \int_{0}^{\infty} \exp \left[-p t-\frac{1}{4 k t}\left(r^{2}+r^{\prime 2}-2 r r^{\prime} \cos \theta\right)^{1 / 2}\right] \frac{d t}{t} \\
& =\frac{1}{2 \pi k} K_{0}\left[q\left(r^{2}+r^{\prime 2}-2 r r^{\prime} \cos \theta\right)^{1 / 2}\right] \\
& = \begin{cases}\frac{1}{2 \pi k} \sum_{n=-\infty}^{\infty} I_{n}(q r) K_{n}\left(q r^{\prime}\right) \cos n \theta, & r<r^{\prime}, \\
\frac{1}{2 \pi k} \sum_{n=-\infty}^{\infty} I_{n}\left(q r^{\prime}\right) K_{n}(q r) \cos n \theta, & r>r^{\prime},\end{cases} \\
& =\sum_{n=-\infty}^{\infty} \bar{u}_{n} \cos n \theta,
\end{aligned}
$$

where the $\bar{u}_{n}$ are given by (20). Also from (18) and (19)

$$
\frac{d^{2} \bar{w}_{n}}{d r^{2}}+\frac{1}{r} \frac{d \bar{w}_{n}}{d r}-\left(\frac{n^{2}}{r^{2}}+q^{2}\right) \bar{w}_{n}=0 .
$$

* We use successively the results, $\$ 6.22,(15)$, and $\$ 11.41,(8)$, of Watson, Theory of Bessel Functions. 
So $\bar{w}_{n}=B_{n}(q) K_{n}(q r)$, where $B_{n}(q)$ is to be determined from the surface condition (3). This requires

Hence, for $r<r^{\prime}$,

$$
-\frac{\partial \bar{v}}{\partial r}+h \bar{v}=0, \quad r=a .
$$

$$
2 \pi k B_{n}(q)\left[h K_{n}(q a)-q K_{n}^{\prime}(q a)\right]=\left[q I_{n}^{\prime}(q a)-h I_{n}(q a)\right] K_{n}\left(q r^{\prime}\right) .
$$

So

$$
\bar{v}_{n}=\bar{u}_{n}+\bar{w}_{n}=(1 / 2 \pi k) K_{n}\left(q r^{\prime}\right) I_{n}(q r)+B_{n}(q) K_{n}(q r), \quad r<r^{\prime} .
$$

Therefore, by the inversion formula, ${ }^{*}$ if $\mu=(\lambda / k)^{1 / 2}$, and $c>0$,

$$
4 \pi^{2} i k v_{n}=\int_{c-i \infty}^{c+i \infty} e^{\lambda t} K_{n}\left(\mu r^{\prime}\right)\left[I_{n}(\mu r)+2 \pi k B_{n}(\mu) K_{n}(\mu r)\right] d \lambda, r<r^{\prime}
$$

and

$$
v=\sum_{n=-\infty}^{\infty} v_{n} \cos n \theta
$$

We have to verify that this value of $v$ satisfies (2); that is, that the $w_{n}$ satisfy (18). From (21) and the inversion formula, we obtain

$$
2 \pi i w_{n}=\int_{c-i \infty}^{c+i \infty} e^{\lambda t} K_{n}(\mu r) B_{n}(\mu) d \lambda,
$$

and this is a continuous function of $t$ for $t \geqq 0$. To show that it vanishes for $t=0$, consider the integral

$$
\int K_{n}(\mu r) B_{n}(\mu) d \lambda
$$

taken round the contour $A B G A$ of Fig. 4, consisting of the line joining $c-i R, c+i R$, and a portion of a circle, center the origin, in the righthand half-plane. There are no poles of the integrand in the contour. $\dagger$ Also, using the asymptotic expansions for the Bessel functions, we find that the integral in (24) round the circular arc tends to zero as $R \rightarrow \infty$. So the integral over the path $c-i \infty, c+i \infty$ vanishes.

6. To reduce (22) to the form (16), consider the integral of the integrand of (22) round the path of Fig. 4 formed by the line $A B$ at distance $c$ from the imaginary axis, the circle $\Gamma$ of radius $R$, the circle $\gamma$ of radius $\epsilon$, and the lines $C D, E F$, on which $\arg \lambda=\pi$, and $-\pi$, respectively. There are no poles of the integrand inside or on the

* Churchill, Mathematische Zeitschrift, vol. 42 (1937), p. 569.

$\dagger$ See the footnote to $\S 6$. 
contour.* The integral round $\gamma$ tends to zero as $\epsilon \rightarrow 0$. Also, using the asymptotic expansions of the Bessel functions, we find that the integral round $\Gamma$ tends to zero as $R \rightarrow \infty$. So in the limit $R \rightarrow \infty, \epsilon \rightarrow 0$,

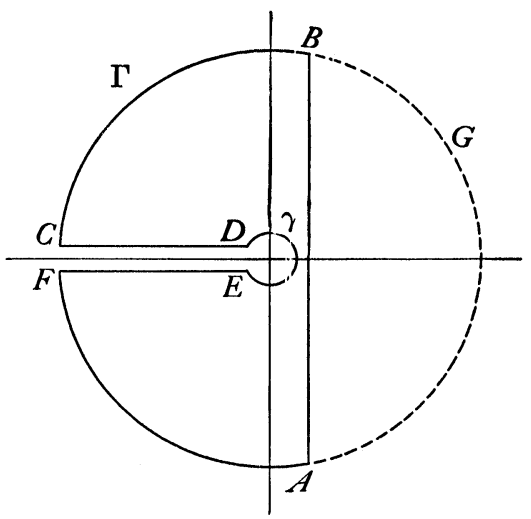

FIG. 4

$v_{n}$ equals the sum of the integrals along $C D$ and $E F$. Putting $\lambda=k \alpha^{2} e^{i \pi}$ in the former, and $\lambda=k \alpha^{2} e^{-i \pi}$ in the latter, we obtain, on reduction, (16).

7. The path $P$ of Fig. 1 in the $\alpha$-plane corresponds to a path in the $\lambda$-plane $\left(\lambda=-k \alpha^{2}\right)$ which starts at infinity where arg $\lambda$ lies between $-\pi$ and $-\pi / 2$, passes to the right of the origin, and ends at infinity, where $\arg \lambda$ lies between $\pi / 2$ and $\pi$. This is the path $L^{\prime}$ of Jeffreys' tract, Operational Methods in Mathematical Physics (2d edition, 1931, p. 29) and not the path $L$ (from $c-i \infty$ to $c+i \infty$ ) as stated by Bromwich in the Proceedings of the Cambridge Philosophical Society, vol. 20 (1921), p. 412. The connection between the two methods employed in this paper is thus clear. There may be an advantage in the former as it seems to give a simpler verification that the expression as found does in fact satisfy all the conditions of the problem.

The University of Sydney and

The University of TASMANia

* To show that $z K_{n}^{\prime}(z)-b K_{n}(z)$, where $b>0$, has no zeros for $R(z) \geqq 0$, we may, since $K_{-n}(z)=K_{n}(z)$, take $n \geqq 0$. That there are no real positive zeros follows from the recurrence formula and the fact that $K_{n}(x)>0$ for real positive $x$. That there are no complex zeros follows from the formula (Gray and Matthews, Treatise on Bessel Functions, 2d edition, 1922, p. 70, (30)): $\left(\lambda^{2}-\mu^{2}\right) \int_{a}^{\infty} x K_{n}(\lambda x) K_{n}(\mu x) d x=$ $a\left[\mu K_{n}(\lambda a) K_{n}^{\prime}(\mu a)-\lambda K_{n}(\mu a) K_{n}^{\prime}(\lambda a)\right], R(\lambda+\mu)>0$. To show there are no pure imaginary zeros $z=i y$, we have $i y K_{n}^{\prime}(i y)-b K_{n}(i y)=\frac{1}{2} \pi i e^{3 n i \pi / 2}\left[-y J_{n}^{\prime}(y)+b J_{n}(y)+i y Y_{n}^{\prime}(y)\right.$ $\left.-i b Y_{n}(y)\right]$, and the real and imaginary parts of this must vanish. This requires $J_{n}(y) Y_{n}^{\prime}(y)-Y_{n}(y) J_{n}^{\prime}(y)=0$, but it is $2 / \pi y$. 